\title{
D-S evidential theory on SEMG signal recognition
}

\section{Weiliang Ding, Gongfa $\mathrm{Li}^{*}$, Ying Sun, Guozhang Jiang and Jianyi Kong}

College of Machinery and Automation,

Wuhan University of Science and Technology,

Wuhan, China

Email: d362667949@163.com

Email: ligongfa@wust.edu.cn

Email: 493530316@qq.com

Email:whjgz@wust.edu.cn

Email: 15697188659@wo.com.cn

*Corresponding author

\section{Honghai Liu}

Intelligent Systems and Biomedical Robotics Group,

School of Computing,

University of Portsmouth,

Portsmouth, PO1 3HE, UK

and

State Key Laboratory of Mechanical System and Vibration,

School of Mechanical Engineering,

Shanghai Jiao Tong University,

Shanghai, China

Email: honghai.liu@sjtu.edu.cn

\begin{abstract}
In order to promote the accuracy and complexity in the recognition of sEMG signals by classifiers, this paper tells a method based on fused D-S evidential theory. Three features are discussed in the choice of parameters, which includes AR model coefficient, cepstral coefficients and time-domain integral absolute value. D-S evidential theory gets information based on information fusion of multi feature sets and multi classifiers. In recognition phase, many groups of data are used for the training and the rest is for the test. Through the compare of the accuracy in different parameters, the result is shown according to the experiment about the data fusion in D-S evidential theory. Six actions are set to be the samples. According to three characters, the recognition accuracy is compared. The result shows that the fused data method of D-S evidential theory has better accuracy and robustness. The further study is to determine the optimal fusion feature set to make more accurate and higher robustness of the classification.
\end{abstract}

Keywords: sEMG signal; D-S evidential theory; fused data; recognition.

Reference to this paper should be made as follows: Ding, W., Li, G., Sun, Y., Jiang, G., Kong, J. and Liu, H. (2017) 'D-S evidential theory on sEMG signal recognition', Int. J. Computing Science and Mathematics, Vol. 8, No. 2, pp.138-145. 


\section{$D-S$ evidential theory on $S E M G$ signal recognition}

Biographical notes: Weiliang Ding received his BS in Measurement and Control Technology and Instrumentation program from Changzhou Institute of Technology, China. He is currently occupied in his MS in Mechanical Design and Theory at Wuhan University of Science and Technology. His current research interests include mechanical $\mathrm{CAD} / \mathrm{CAE}$, signal analysis and processing.

Gongfa Li received his $\mathrm{PhD}$ degree in Wuhan University of Science and Technology, Wuhan, China. He is currently a Professor in Wuhan University of Science and Technology. His major research interests are computer aided engineering, mechanical CAD/CAE, modelling and optimal control of complex industrial process.

Ying Sun is currently an Associate Professor in Wuhan University of Science and Technology. Her major research focuses on teaching research in mechanical engineering.

Guozhang Jiang received his $\mathrm{PhD}$ degree in Wuhan University of Science and Technology, China. He is currently a Professor in Wuhan University of Science and Technology. His research interests are computer aided engineering, mechanical CAD/CAE and industrial engineering and management system.

Jianyi Kong received his $\mathrm{PhD}$ degree in Helmut Schmidt Universitat, Germany. $\mathrm{He}$ is currently a Professor in Wuhan University of Science and Technology. His research interests are intelligent machine and controlled mechanism, mechanical and dynamic design and fault diagnosis of electrical system, mechanical CAD/CAE, intelligent design and control.

Honghai Liu received his $\mathrm{PhD}$ in Intelligent Robotics from Kings College, University of London, London, UK. He is currently a Professor of Intelligent Systems in Portsmouth University, Portsmouth, UK. His research interests are approximate computation, pattern recognition, multi-sensor-based information fusion and analytics, human machine systems, advanced control, intelligent robotics and their practical applications.

\section{Introduction}

Recently, the research on bioelectric signal has aroused wide attention. As one of the significant signal, surface electromyogram (sEMG) signal is applied in many fields, such as clinical medicine, sports medicine and rehabilitation engineering. The recognition of sEMG signal is proved to be meaningful in its application (Arjunan and Kumar, 2007).

With the progress of neurophysiology, researchers have a fully understanding of the mechanism of physiological electromyography (Coatrieux et al., 1983). In the process of muscle contraction which caused by the brain excitation, different excitations result in the different frequency of electric pulses, causing the different numbers of myofibril contraction (Frigo et al., 2000). And in different patterns of muscle movements, the use of muscle is also different. EMG signal collected in the detection electrode is the sum of electric potential caused by each myofibril's action in the detection points (Hudgins et al., 1993). Therefore, it is known that different muscle movement patterns are produced by different muscle groups, and accordingly, the sEMG signal is different (Saridis and Gootee, 1982). It is possible to find the corresponding muscle movement 
patterns from different sEMG signals. In fact, the recognition of sEMG signal is pattern recognition (Khalil and Duchene, 2000). Pattern always contains the distribution information of time and space. The vital parts of the typical pattern recognition system are the feature extraction and classifier (Lee and Saridis, 1982). A basic recognition system is shown in Figure 1.

Figure 1 Basic recognition system

\begin{tabular}{|c|c|c|}
\hline $\begin{array}{c}\text { Signal Acquisition } \\
\text { and Pre-processing }\end{array}$ & $\longrightarrow$ Feature Extraction & Classifier \\
\cline { 1 - 1 }
\end{tabular}

As it is shown above, the basic recognition system consists of three parts, which are signal acquisition and pre-processing, feature extraction and classifier (Lee et al., 1997).

The role of signal acquisition system is to transfer the object's characteristics into the calculating information of recognition system. At present, in the related study, there is a way to encode the sEMG signal by channels, such as single channel, binary channels, four channels and even more channels (Liu et al., 2005).

The amount of information obtained through the acquisition is often relatively large, so the characteristics of different objects could not be compared and determined. In order to realise the classification and recognition effectively, the extraction of collected information is needed to get a set of information which reflects the characteristics of the object. And feature vectors are used to represent this pattern:

$$
\lambda=\left[\lambda_{1}, \lambda_{2} \ldots \lambda_{i}\right]
$$

Among $i$ refers to the dimension of feature vectors.

Classifier is a kind of decision tool, which uses different algorithms to classify characteristics. At present, in the study about the recognition of sEMG signals, Bayesian decision, maximum likelihood classifier, clustering method, artificial neural network and so on have a wide range of applications. Among them, the artificial neural network is the most used one.

In this paper, the D-S evidential theory is applied to the sEMG recognition. According to the contrast of three feature sets and fusion data, the D-S evidential theory is proved to be more efficient and accurate in sEMG signal recognition. The most efficient and practical method in collecting sEMG signals should be based on the accuracy and real-time performance. The usage of D-S evidential theory in the SEMG recognition is purposed on getting more accurate data in the shortest time (Zhou and Rymer, 2003).

\section{D-S evidential theory}

In studying the sEMG pattern recognition system, researchers have proposed many kinds of representation methods, such as zero crossing point in time domain, absolute value integral in time domain; transform coefficients in time-frequency domain, AR model coefficient (ARMC), variance, complexity of the nonlinear dynamics and fractal dimension (Binaghi et al., 2000). But sEMG signal is a physiological signal with poor reproducibility, sometimes even contradictory. A feature set only describes signals from a 
point of view $(\mathrm{Ca}$ and $\mathrm{Li}, 2002)$. It is difficult to find a characteristic parameter sets to perfectly identify different actions. Therefore, the practical application of the above methods in multi DOF prosthetic or hand still has a certain distance. Also, signal with different type of features reflect different properties of the target (Song et al., 2014). It is difficult to form a complete understanding of the essential characteristic of the target (Ni et al., 2015). The fusion of different signal features represents the original target more fully and completely. Therefore, researchers usually use structured neural network to fuse multiple feature set parameters to further improve the accuracy and robustness of sEMG action recognition. But the disadvantage is that the fusion of multiple features set parameters leads to the quantity of computation ( $\mathrm{Lv}$ et al., 2016). It takes more processing time. In this paper, the decision layer fusion method is proposed. The feature vector of each set is trained and classified by neural network classifier. Each single classifier the classification results as a body of evidence. In the same frame of discernment, different evidence bodies synthesise into a new body of evidence, and the reuse of certain decision rules from the new evidence makes motion discrimination. This body of evidence can be achieved through a variety of merger rules, such as the majority voting, fuzzy logic, decision template and D-S evidence theory (He et al., 2001). This paper uses D-S evidence theory to achieve the integration of decision making.

\section{Methodology}

D-S evidence theory discusses a 'recognition framework' (frame of discernment), so the possible recognition results or assumptions about the independence of propositions is defined within this framework (set). The set of all possible subsets contained is called a power set of $\theta$, represented by $\Omega(\theta)$. In gesture recognition, if the class of the sample is identified as $x, y, z$ in this case, the 'identification framework' and 'power set' are defined as follows:

$$
\begin{aligned}
& \theta=(x, y, z) \\
& \Omega(\theta)=\{\phi,\{x\},\{y\},\{z\},\{x, y\},\{x, z\},\{y, z\},\{x, y, z\}\}
\end{aligned}
$$

Among $\phi$ refers to the situation that the answer is not $x, y$ or $z$. It can be other types. The subset refers to the answer could be $x$ or $y$. It is similar with other subsets. It can be seen that when there are $N$ elements in $\theta$, there are $2^{N}-1$ elements in $\Omega(\theta)$.

For the defined 'recognition framework' $\theta$, power set function can be defined as a mapping from $\Omega(\theta)$ to $[0,1]$, which satisfies two conditions:

$$
\begin{aligned}
& m: \Omega(\theta) \rightarrow[0,1] \\
& m(\varnothing) \neq 0, \quad \sum_{A \subseteq \Omega(\theta)} m(A)=1
\end{aligned}
$$

Among $m$ is a basic probability assignment function. When $A=\theta, m(A)$ means that it is confused in distributing $m$. When $A$ is a subset of $\theta$ and $m(A) \neq 0$, it is a focal function of $m$.

If $\theta$ is a 'recognition framework', $m: \Omega(\theta) \rightarrow[0,1]$ is the basic probability assignment function in frame $\mathrm{Bel}$, so the belief function is defined as: 


$$
\operatorname{Bel}: \Omega(\theta) \rightarrow[0,1], \operatorname{Bel}(A)=\sum_{B \subset A} m(B)
$$

Plausibility function $P l s$ is defined as:

$$
P l s: \Omega(\theta) \rightarrow[0,1], P l s(A)=1-B e(\bar{A})=\sum_{B \cap A=\varnothing} m(B)
$$

Belief function $\mathrm{Bel}$ means a measure that proposition $A$ determines to be set. Plausibility function $\mathrm{Pls}$ means an uncertainty proposition that $A$ probably determines to be set.

Figure 2 Belief internal

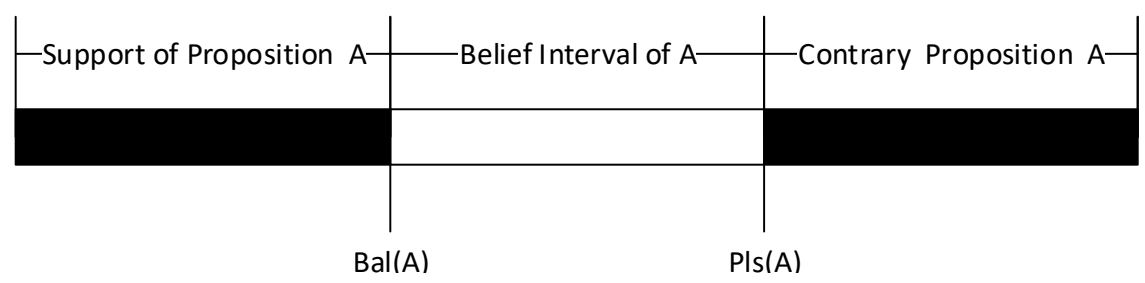

If the belief function $\operatorname{Bel}(A)$ and plausibility function $P l s(A)$ of proposition $A$ are known, the belief interval of $A$ is $[\operatorname{Bel}(A), P l s(A)]$, which is shown in Figure 2. This interval represents the uncertain probability of the occurrence of $A$. The lower bound is the belief of proposition, which means the minimal probability that proposition, occurs based on the direct evidence of the sensor. The upper bound is the plausibility of proposition, which means the combination of the belief of proposition and the potential possibility of proposition occurred. So the bound can explain how much of evidence supports the proposition, and how much is not known about the proposition, and how much it is determined to refute the proposition.

This is a hypothesis that $\mathrm{Bel}_{1}$ and $\mathrm{Bel}_{2}$ are belief functions from two types of sensors in the same 'recognition framework' $\theta . m_{1}$ and $m_{2}$ are the corresponding basic probability assignment functions. If focal functions of $B e l_{1}$ and $B e l_{2}$ are $A_{1}, A_{2}, \ldots, A_{K}$, and $B_{1}$, $B_{2}, \ldots, B_{K}$. According to the $\mathrm{D}-\mathrm{S}$ orthogonal principle, the composed basic probability assignment function $m: \Omega(\theta) \rightarrow[0,1]$ is shown in following formula.

$$
\begin{array}{ll}
m(C)= \begin{cases}0, & C=\varnothing \\
\frac{\sum_{A_{i} \cap B_{j}=C} m_{1}\left(A_{i}\right) m_{2}\left(B_{j}\right)}{1-K}, & C \neq \varnothing\end{cases} \\
K=\sum_{A_{i} \cap B_{j}=C} m_{1}\left(A_{i}\right) m_{2}\left(B_{j}\right)
\end{array}
$$

According to the above belief function, it can be seen that belief degrees in each evidences are the same. But after the study on the module of multi sensors recognition system, it is easy to find that each sensor has unique perceptive precision, so belief degrees in each evidence are not exactly the same. The rules of evidence combination need to be improved. That means that weighted rules are used for fusion detection.

In the fusion of results detection, the selection of weights is very important. For the belief degree, it usually takes the performance of sensor in similar conditions as evidence. 


\section{Experimental simulation}

In order to verify the classification performance of information fusion system, sEMG signals of six hand movements are collected to take the experiment. Four pairs of differential electrodes are sequentially disposed in the flexor carpi ulnaris, the musculus flexor digitorum sublimis, the palmaris longus tendon and the flexor carpi radialis, which simultaneous collect four channels of sEMG signals. The healthy subjects complete the 300 groups of spherical gripping, cylindrical gripping, thumb index finger pinching, three finger pinching, lateral pinching and four fingers lifting. Six actions are shown in Figure 3. Each action should have 50 groups of data. The mean value of collected signals should be processed. In many kinds of sEMG features, the following three kinds of sEMG feature parameters are extracted as the input feature vectors of the single classifier, which are ARMC, cepstral coefficients (CCs) and time-domain integral absolute value (TDIAV). Samples images are shown in Figure 3.

Figure 3 Collected sample images (see online version for colours)

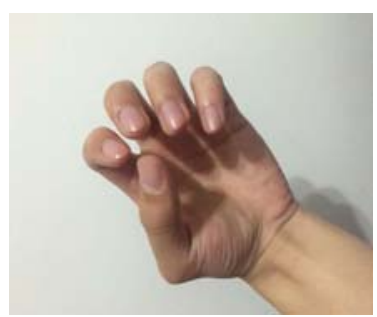

(a)

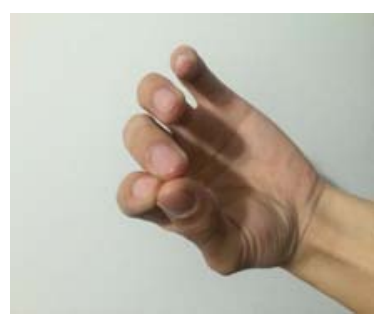

(d)

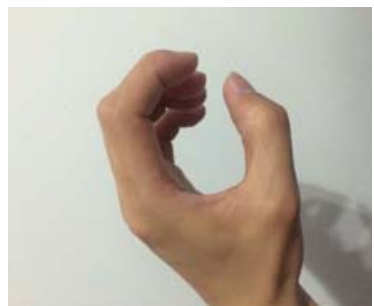

(b)

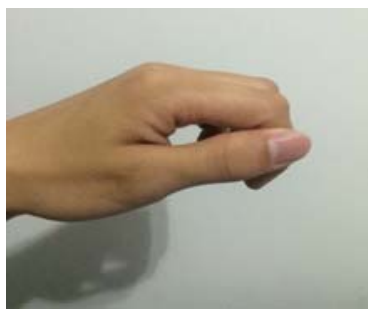

(e)

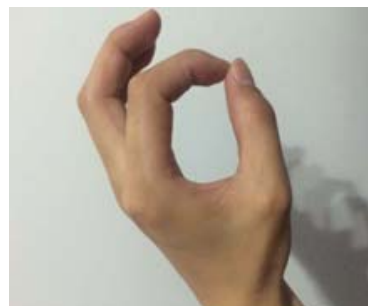

(c)

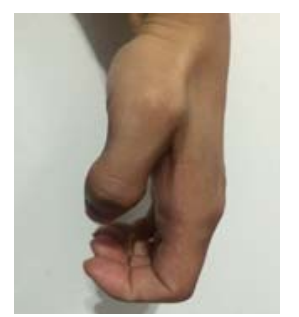

(f)

In the experiment, 30 randomly records from each type of action samples are selected as the training set of $3 \mathrm{BP}$ neural network classifiers. And the residual signals are as the test set. Three feature sets are extracted from four channel signals and construct the normalised feature vectors. Structures of 3 BP neural network respectively are $24 \times 12 \times 6$ (ARMC, CCs) and $28 \times 14 \times 6$ (TDIAV). For all kinds of action in training samples, the value of the corresponding output node sets 1 and others are 0 . They are expected output in training. When the training error is less than 0.01 , the network is convergence. At the same time, the learning rate is 0.01 . When network tests, if an output node is greater than 0.5 , and the rest output nodes are less than 0.5 , the action can be distinguished as the corresponding action mode of this node. Otherwise, it is rejected to be the corresponding mode. The identification rates of classification about 120 groups of data from three different feature sets are shown in Table 1. 
Table 1 Identification rates of classification

\begin{tabular}{lccccc}
\hline$N W$ & $S s$ & $C N s$ & $E N s$ & $R N s$ & Recognition rate \\
\hline ARMC & 120 & 104 & 11 & 5 & $86.7 \%$ \\
TDIAV & 120 & 94 & 19 & 7 & $78.3 \%$ \\
CCs & 120 & 101 & 13 & 6 & $84.1 \%$ \\
$\alpha 0.00$ & 120 & 114 & 6 & 0 & $95.0 \%$ \\
$\alpha 0.80$ & 120 & 113 & 0 & 7 & $94.2 \%$ \\
$\alpha 0.91$ & 120 & 111 & 0 & 9 & $92.5 \%$ \\
\hline
\end{tabular}

\section{Conclusions and future work}

According to the physiological characteristics of sEMG signal, this paper proposes a classification method based on information fusion of multi feature sets and multi-classifiers (Stoykov et al., 2005). In these selected feature sets, the classification accuracy of time domain integral is the lowest, while the ARMC has the highest accuracy in classification. The selection of the three feature sets is due to its good classification accuracy, low computational complexity and constringency stability. With the combination of these characters, they completely and complementary reflect the different motion modes. After the evidence accumulated, results of three classification feature set are fused without uncertainty and imprecision. The accuracy of fused classification is better than single feature classification result. In the classification, the rejected data are set to provide the user of the prosthetic hand security (Xia et al., 2014). The direction of future work is to further determine the optimal fusion feature set to make more accurate and higher robustness of the classification (Zhang et al., 2014).

\section{Acknowledgements}

This work was supported by grants of National Natural Science Foundation of China (Grant Nos. 51575407, 51575338, 1575412, 61273106).

\section{References}

Arjunan, S. and Kumar, D.K. (2007) 'Recognition of facial movements and hand gestures using surface electromyogram (sEMG) for HCI based applications', Biennial Conference of the Australian Pattern Recognition Society on Digital Image Computing Techniques \& Applications, pp.1-6.

Binaghi, E., Gallo, I. and Madella, P. (2000) 'A neural model for fuzzy Dempster-Shafer classifiers', International Journal of Approximate Reasoning, Vol. 25, No. 2, pp.89-121.

$\mathrm{Ca}$, S. and Li, K. (2002) 'Relationships between combination methods and measures of diversity in combining classifiers', Information Fusion, Vol. 3, No. 2, pp.135-148.

Coatrieux, J.L., Cordella, F. and Barone, R. (1983) 'Automatic classification of electromygraphic signals', Electroencephalography \& Clinical Neurophysiology, Vol. 55, No. 55, pp.333-341. 
Frigo, C., Ferrarin, M., Frasson, W., Pavan, E. and Thorsen, R. (2000) 'EMG signals detection and processing for on-line control of functional electrical stimulation', Multimedia Tools and Applications, Vol. 10, No. 8, pp.351-360.

He, Y., Chu, F. and Zhong, B. (2001) 'A study on group decision-making based fault multi-symptom-domain consensus diagnosis', Reliability Engineering System Safety, Vol. 74, No. 1, pp.43-52.

Hudgins, B., Parker, P. and Scott, R.N. (1993) 'A new strategy for multifunction myoelectric control', IEEE Transactions on Biomedical Engineering, Vol. 40, No. 1, pp.82-94.

Khalil, M. and Duchene, J. (2000) 'Uterine EMG analysis: a dynamic approach for change detection and classification'. IEEE Transactions on Biomedical Engineering, Vol. 47, No. 6, pp.748-756.

Lee, S. and Saridis, G.N. (1982) 'The control of a prosthetic arm by EMG pattern recognition', IEEE Transactions on Automatic Control, Vol. 29, No. 4, pp.336-344.

Lee, S.P., Kim, J.S. and Park, S.H. (1997) 'An enhanced feature extraction algorithm for EMG pattern classification', IEEE Transactions on Rehabilitation Engineering: A Publication of the IEEE Engineering in Medicine \& Biology Society, Vol. 4, No. 4, pp.439-443.

Liu, H., Chen, X. and Chen, Y. (2005) 'Wavelet transform analyzing and real-time learning method for EMG signal in motion discrimination', International Conference on Neural Interface \& Control, Vol. 13, No. 1, pp.127-130.

Lv, L., Han, L., Fan, T. and Zhao, J. (2016) 'Artificial bee colony algorithm with accelerating convergence', International Journal of Wireless and Mobile Computing, Vol. 10, No. 1, pp.76-82.

Ni, J., Xu, X., Ding, S. and Sun, T. (2015) 'An adaptive extreme learning machine algorithm and its application on face recognition', International Journal of Computing Science and Mathematics, Vol. 6, No. 6, pp.611-619.

Saridis, G.N. and Gootee, T.P. (1982) 'EMG pattern analysis and classification for a prosthetic arm', IEEE Transactions on Biomedical Engineering, Vol. 29, No. 6, pp.403-412.

Song, X-L., Song, Z-Y. and Liu, C-Y. (2014) 'Heating exchange process PIDNN control system research based on T-S fuzzy model', International Journal of Wireless and Mobile Computing, Vol. 7, No. 1, pp.94-101.

Stoykov, N.S., Lowery, M.M., Heckman, C.J., Taflove, A. and Kuiken, T.A. (2005) 'Recording intramuscular EMG signals using surface electrodes', International Conference on Rehabilitation Robotics, IEEE.

Xia, X., Wei, B. and Xie, C. (2014) 'An improved PSO with detecting and local-learning strategy', International Journal of Computing Science and Mathematics, Vol. 5, No. 4, pp.370-380.

Zhang, G-Y., Zeng, J-C. and Xue, S-D. (2014) 'Research on task allocation of multi-target search with swarm robots', International Journal of Wireless and Mobile Computing, Vol. 7, No. 3, pp.297-304.

Zhou, P. and Rymer, W.Z. (2003) 'Motor unit action potential number estimation in the surface electromyogram, wavelet matching method and its performance boundary', Proceeding of the 1st international IEEE EMBS Conference on Neural Engineering Capri Island, pp.20-22. 